\title{
The faint X-ray source population near 3C 295
}

\author{
V. D’Elia ${ }^{1}$, F. Fiore ${ }^{1}$, M. Elvis ${ }^{2}$, M. Cappi ${ }^{3}$, S. Mathur ${ }^{4}$, P. Mazzotta $^{5}$, E. Falco ${ }^{6}$, and F. Cocchia ${ }^{1}$ \\ 1 INAF - Osservatorio Astronomico di Roma, via Frascati 33, 00040 Monteporzio-Catone (RM), Italy \\ ${ }^{2}$ Harvard-Smithsonian Center for Astrophysics, Cambridge, MA 02138, USA \\ ${ }^{3}$ IASF - CNR, Bologna, Italy \\ 4 The Ohio State University, Columbus, OH 43210, USA \\ 5 University of Durham, South Road, Durham DH1 3LE, UK \\ ${ }^{6}$ Smithsonian Institution Whipple Observatory, AZ 85645, USA
}

Received 10 June 2003 / Accepted 31 March 2004

\begin{abstract}
We present a statistical analysis of the Chandra observation of the source field around the 3C 295 galaxy cluster $(z=0.46)$ to search for clustering of X-ray sources. We applied three different methods of analysis, all suggesting a strong clustering in the field on scales of a few arcmin. In particular 1) the $\log N-\log S$ computed separately for the four ACIS-I chips reveals that there is a significant $(3.2 \sigma$ in the $0.5-2 \mathrm{keV}, 3.3 \sigma$ in the $2-10 \mathrm{keV}$ and $4.0 \sigma$ in the $0.5-10 \mathrm{keV}$ band) excess of sources to the North-North East and a void to the South of the central cluster; 2) the two point, two-dimensional Kolmogorov-Smirnov (KS) test, shows the probability that the sources are uniformly distributed is only a few percent; 3) a strong spatial correlation emerges from the study of the angular correlation function of the field: the angular correlation function (ACF) shows a clear signal on scales of $0.5 \div 5 \mathrm{arcmin}$, correlation angle in the $0.5-7 \mathrm{keV}$ band $\theta_{0}=8.5_{-4.5}^{+6.5}, 90 \%$ confidence limit (assuming a power law ACF with slope $\gamma=1.8$ ). This correlation angle is 2 times higher than that of a sample of 8 ACIS-I field at the $2.5 \sigma$ confidence level. The above scales translate to $0.2 \div 2 \mathrm{Mpc}$ at the cluster redshift, higher than the typical cluster core radius, and more similar to the size of a "filament" of the large scale structure.
\end{abstract}

Key words. X-rays: galaxies - galaxies: clusters: individual: 3C 295 - X-rays: diffuse background cosmology: large-scale structure of Universe

\section{Introduction}

$N$-body and hydrodynamical simulations show that clusters of galaxies lie at the nexus of several filaments of galaxies (see e.g., Peacock 1999; Davé et al. 2001, and references therein). Such filaments map out the "cosmic web" of voids and filaments of the large scale structure (LSS) of the Universe. According to the same simulations these filaments contain a large fraction (30-40\%) of the baryons in the Universe at $z<1$ (the remainder ending up in the hot gas in clusters of galaxies, and in stars and cold gas clouds). Despite its larger total mass, observations of the intergalactic matter in filaments so far have yielded only limited information, mostly due to its low density (most of the baryons in this phase should be at densities only 10-100 times higher than the average density of the Universe). The most direct way to detect a filament at low redshift is through its soft X-ray diffuse emission (see e.g., Zappacosta et al. 2002; Soltan et al. 2002), or through soft X-ray and UV absorption line studies (see e.g., Fiore et al. 2000, and references therein; Nicastro et al. 2002, 2003; Mathur et al. 2003). Both methods require very difficult observations, at

Send offprint requests to: V. D'Elia, e-mail: delia@mporzio.astro.it the limit of the present generation of X-ray and UV facilities. Alternatively, filaments could be mapped out by galaxies (Daddi et al. 2001; Giavalisco \& Dickinson 2001) and by the much more luminous Active Galactic Nuclei (AGNs), assuming that AGNs trace galaxies. Since rich clusters of galaxies are good indicators of regions of sky where filaments converge, numerous AGN searches around clusters of galaxies have been performed in the past. Several of these studies suggest overdensities of AGNs around distant clusters of galaxies (Molnar et al. 2002, for the cluster Abell 1995; Best et al. 2002, for MS1054-03; Martini et al. 2002, for Abell 2104; Pentericci et al. 2002, for the protocluster at $z \sim 2.16$ around the radio galaxy MRC 1138-206; see also Almaini et al. 2003, for the ELAIS North field). Many of these studies have been performed in X-rays, since extragalactic X-ray sources, which are mostly AGNs, have a space density $\sim 10$ times higher than optically selected AGNs (see Yang et al. 2003), and therefore provide denser tracing of LSS. One of the first studies of the X-ray source population in cluster fields was performed by Cappi et al. (2001) who studied the Chandra $8 \times 8$ arcmin ACIS fields around the distant cluster of galaxies RX J003033.2+261819 $(z=0.5)$ and 3C $295(z=0.46)$. Cappi et al. (2001) reported the tentative detection of an overdensity of faint X-ray 
sources in a region of a few arcmin around both clusters, with respect to the average X-ray source density at the same flux limit. However, the observations were too short ( $\sim 30 \mathrm{ks}$ and $\sim 18 \mathrm{ks}$, respectively), and the source samples consequently too small, to derive any more detailed conclusion.

In addition to counting sources in selected sky areas, the clustering of X-ray sources can be studied using other observational and statistical tools. Gilli et al. (2003) report narrow spikes in the redshift distribution of the sources in the Chandra Deep Field South (CDFS), indicating strong clustering of sources in these narrow redshift ranges. If the source samples are sufficiently large (of the order of 100 sources or more) one derives more detailed and quantitative information on the source clustering by studying the angular correlation function (ACF) of the sources in the field. Vikhlinin \& Forman (1995) were among the first to study the ACF in the X-ray band; they evaluated an average ACF from a large set of deep ROSAT observations, covering in total $40 \mathrm{deg}^{2}$ of sky. They found positive correlation on scales from a fraction of arcmin to tens of arcmin. However, their best fit "correlation angle" $\theta_{0} \sim 10$ arcsec, is smaller than the ROSAT PSPC Point Spread Function ( $\sim 25$ arcsec FWHM on-axis); as noted by the authors, this leads to an "amplification bias" and their measured ACF is consequently somewhat overestimated.

This effect can be greatly mitigated by using a telescope like Chandra, whose on-axis PSF is only $0.5 \operatorname{arcsec} F W H M$, 50 times better than the PSPC on-axis PSF. So far a correlation analysis of Chandra X-ray sources has been published only by Giacconi et al. (2000), who used the first $100 \mathrm{ks}$ of observation of the CDFS, and by Yang et al. (2003) who analyzed a mosaic of 9 moderately deep ( $30 \mathrm{ks}$ ) Chandra pointings of the Lockman Hole area. Yang et al. studied the source angular correlation on scales of several tens of arcmin, being limited on smaller scales by the small number of detected sources per unit area.

Our main goal in this paper is to push this kind of analysis toward a) smaller scales, of order of a few arcmin; and b) targeting a field where the likelihood of observing LSS is high, i.e. a field around a cluster of galaxies. For these reasons we again observed the 3C 295 field with Chandra for about $100 \mathrm{ks}$, where Cappi et al. (2001) found tentative evidence of an overdensity of faint X-ray sources in a region of a few arcmin around the central cluster.

The paper is organized as follows: Sect. 2 presents the observations and data reduction; Sect. 3 presents the results of our analysis; Sect. 4 discusses such results and draws our conclusions.

\section{Chandra observations and data reduction}

Chandra observed the $16^{\prime} \times 16^{\prime}$ field around the 3C 295 cluster with ACIS-I (Garmire 1997) on May 18, 2001. The target of the observation is located at the position of 3C $295 \alpha=14: 11: 10$, $\delta=+52: 13: 01$ (J2000) and exposure time is $\sim 92 \mathrm{ks}$. The data reduction was carried out using the Chandra Interactive Analysis of Observations (CIAO) software version 2.1.3 (http://cxc.harvard.edu/ciao). No strong background flares are present in the observation. The data analysis has been performed separately in the soft $0.5-2 \mathrm{keV}$ band, in the hard 2-7 keV band and in the broad $0.5-7 \mathrm{keV}$ band. Since the ACIS background rises sharply above $\sim 7 \mathrm{keV}$, we excluded all data with $E>7 \mathrm{keV}$.

To check our analysis method, an identical analysis was performed for the Chandra Deep Field South (CDFS, see Giacconi et al. 2002) in the $0.5-2 \mathrm{keV}$ and $2-7 \mathrm{keV}$ bands. The CDFS is a collection of 11 separate ACIS-I observations centered on the same point, $\alpha=03: 32: 28.0, \delta=$ $-27: 48: 30$ (J2000), but with different roll angles. For this reason, the effective exposure time changes in different regions of the field. For the CDFS analysis we selected only the region with the maximal effective exposure time, which is $\sim 942 \mathrm{ks}$; the region is slightly smaller than the full 3C 295 field.

In the following subsection the source detection procedure is described, while Sect. 2.2 deals with the sky coverage evaluation, which will be used in Sect. 3 for the $\log N-\log S$ calculation.

\subsection{Source detections}

The source detection was carried out using the "PWDetect" algorithm. This is a wavelet-based source detection code for Chandra X-ray data developed at the Osservatorio Astronomico di Palermo (Damiani et al. 1997a,b). PWDetect performs a multiscale analysis of the data, allowing detection of both pointlike and moderately extended sources in the entire field of view. This detection algorithm was compared with other detection tools, namely, the CIAO detection tool CELLDETECT and the Ximage DETECT. The latter algorithms detected a smaller number of sources than PWDetect in both the CDFS and 3C 295 field, and in all energy bands. In addition, they tended to find extended sources as multiple detections.

PWDetect requires a Chandra FITS event file and the associated exposure map as inputs. The exposure maps were computed using the CIAO task MKEXPMAP for each ACIS-I chip and then combined together into the single exposure map required by PWDetect for each of the $0.5-2 \mathrm{keV}, 2-7 \mathrm{keV}$ and $0.5-7 \mathrm{keV}$ energy bands. The sources were detected using minimum and maximum wavelet scales of 0.5 and 16 arcsec, respectively. We assumed a probability detection threshold of $2 \times 10^{-5}$. Since the local background level is of the order of $0.1 \mathrm{cts} / \operatorname{arcsec}^{2}$, the minimum number of counts for a source to be detected is $\sim 7$. The assumed probability and the number of independent cells used by PWdetect give the expected number of spurious sources per field to be $<1$ in all energy bands. We identified 89 sources in the $0.5-2 \mathrm{keV}$ band lying within the flux range $3.2 \times 10^{-16}-4.4 \times 10^{-13} \mathrm{erg} \mathrm{cm}^{-2} \mathrm{~s}^{-1}, 71$ sources in the $2-7 \mathrm{keV}$ band within the flux range $1.7 \times 10^{-15}-5.8 \times 10^{-14} \mathrm{erg} \mathrm{cm}^{-2} \mathrm{~s}^{-1}$ and 121 sources in the $0.5-7 \mathrm{keV}$ band in the flux range $8.8 \times 10^{-16}-1.2 \times 10^{-13} \mathrm{erg} \mathrm{cm}^{-2} \mathrm{~s}^{-1}$. The central cluster was not considered in the analysis, thus we excluded the detections in the $\sim 2^{\prime} \times 2^{\prime}$ central region. The counts in the $0.5-2 \mathrm{keV}, 2-7 \mathrm{keV}$ and $0.5-7 \mathrm{keV}$ bands were converted in $0.5-2 \mathrm{keV}, 2-10 \mathrm{keV}$ and $0.5-10 \mathrm{keV}$ fluxes, respectively, by multipling the counts by the conversion factors 
Table 1. Observed optical magnitudes in the $R$ band of the sources in Fig. 2.

\begin{tabular}{lc}
\hline \hline Sources & $R_{\mathrm{mag}}$ \\
\hline $\mathrm{A}$ & 18.8 \\
$\mathrm{~B}$ & 18.15 \\
$\mathrm{C}$ & 19.11 \\
$\mathrm{D}$ & 17.95 \\
$\mathrm{E}$ & 18.18 \\
$\mathrm{~F}$ & 19.11 \\
\hline
\end{tabular}

$5.65 \times 10^{-12}, 2.70 \times 10^{-11}$ and $1.24 \times 10^{-11}$ (from PIMMS: http://heasarc.gsfc.nasa.gov/Tools/w3pimms.html). These factors are appropriate for a $\Gamma=1.8$ power law spectrum with a Galactic absorption toward the 3C 295 field of $N_{\mathrm{H}}=1.33 \times 10^{20} \mathrm{~cm}^{-2}$ (Dickey \& Lockman 1990), and take into account the quantum efficiency degradation of the ACIS chips (http://asc . harvard.edu/cal/Acis).

Figure 1 shows the 3C 295 field in the first two bands; the detected sources are indicated by circles. The larger circle in the upper left chip of the $0.5-2 \mathrm{keV}$ picture indicates an extended source (detected on a 16 arcsec scale, see next subsection) which is probably a group or a cluster of galaxies. A close observation of this region shows other count peaks which could be other X-ray sources, possibly the galaxies in the group, below the detection threshold, although they may be spurious detections caused by positive fluctuations of the surface brightness.

Visually the sources give a strong impression of being concentrated to the East, and expecially the NE. This is the same trend reported in Cappi et al. (2001). We will proceed to quantify this impression in the following sections.

\subsection{Extended sources}

As mentioned before, in the soft and broad band, we identified an extended source on a scale of 16 arcsec, which could also be two or more confused point sources. This source is marked in Fig. 1 by a large circle and its position is RA $=14: 11: 37$, Dec $=+52: 18: 35$; its $0.5-2 \mathrm{keV}$ flux is $4.4 \times 10^{-15} \mathrm{erg} \mathrm{cm}^{-2} \mathrm{~s}^{-1}$ and the $0.5-7 \mathrm{keV}$ one is $1.1 \times 10^{-14} \mathrm{erg} \mathrm{cm}^{-2} \mathrm{~s}^{-1}$. In Fig. 2 we show the contours of the $0.5-7 \mathrm{keV}$ image superimposed on the image taken at the TNG with the DOLORES camera on March 19, 2002, with an exposure time of $900 \mathrm{~s}$; the seeing was 1.3 arcsec and the limiting magnitude $R_{\text {lim }}=24.1$. The southern strong X-ray emitter, which is a pointlike source, has no optical counterpart down to the limiting magnitude of the observation, while the extended emission is centered on two bright $R_{\text {mag }} \sim 18.5$ galaxies. Table 1 reports the magnitudes of the galaxies marked with capital letters in Fig. 2.

If we assume that these galaxies are at a $z$ similar to that of $3 \mathrm{C} 295, z=0.46$, then their absolute magnitude is $M_{B} \simeq-23$. This is almost two orders of magnitude more luminous than the break magnitude of the galaxy luminosity function at $z=0.5$, that is $M_{B}^{*}=-21.3$ (e.g., Poli et al. 2003). Moreover, we note that the central radio galaxy of the 3C 295 cluster has $R_{\text {mag }}=18.4$ in our image, while the X-ray
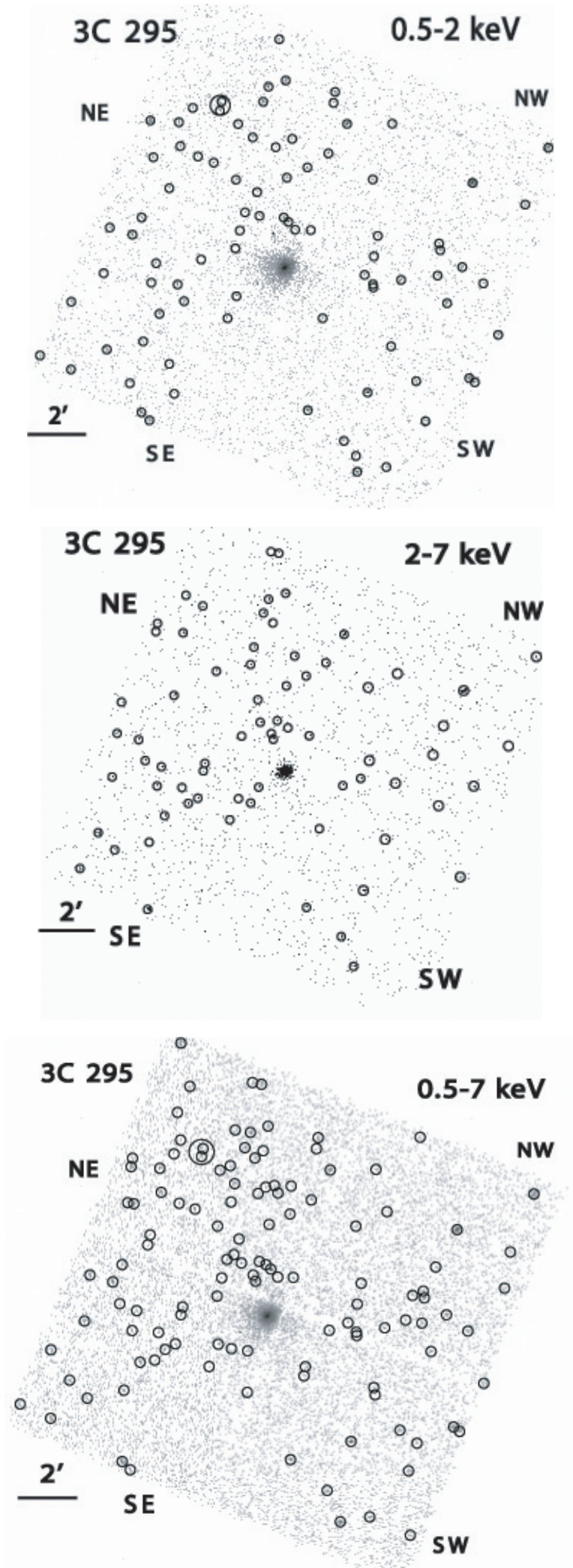

Fig. 1. The Chandra 3C 295 field in the $0.5-2 \mathrm{keV}$ band (top panel), in the $2-7 \mathrm{keV}$ band (central panel) and in the $0.5-7 \mathrm{keV}$ band (bottom panel). Circles represent the sources detected with the wavelet-based source detection code "PWdetect". The largest circles in the top and bottom panels indicates a very extended source, possibly a group of galaxies. The brightest source in the center of the field is the cluster of galaxies 3C 295. 


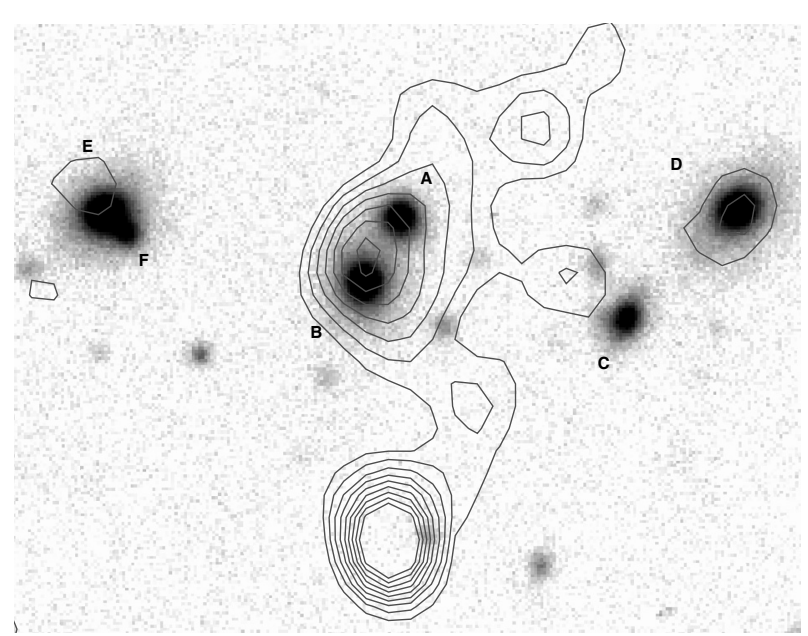

Fig. 2. The contours of the $0.5-7 \mathrm{keV}$ band image overimposed to the optical ( $R$ band) image of the extended source identified in the soft and broad band. Capital letters are associated with galaxies whose $R$ observed magnitudes are reported in Table 1 . The image width is $\sim 1.25$ arcmin.

flux of the 3C 295 cluster is $\sim 1.0 \times 10^{-12} \mathrm{erg} \mathrm{cm}^{-2} \mathrm{~s}^{-1}$ in the 0.5-4.5 keV band (see e.g., Henry \& Henriksen 1986). We conclude that it is unlikely that the optical sources corresponding to the extended X-ray emission are located at $z=0.46$. Since the extended source is not detected in the $2-7 \mathrm{keV}$ band, we can only put an upper limit on the temperature of the putative cluster or group; using a Raymond-Smith model with a solar abundance ratio of 0.4 , we find $T<3 \mathrm{keV}$ if we assume the same redshift of 3C 295 and $T<2 \mathrm{keV}$ for $z=0$. On the other hand, an upper temperature of $2 \div 3 \mathrm{keV}$ poses an upper limit on the luminosity and thus on the redshift; unfortunately, this limit is not significant for our purposes.

We checked for other signs of diffuse emission in the 3C 295 field, and in particular in the NE region, where the detected sources appear to be concentrated. To do this, we subtracted the detected sources from the image and we computed the average background level of the observation in the 0.5-7 keV band; the central part of the image was excluded because of the high level of counts due to the central cluster. Next, we compared this background level with the counts evaluated in 25 regions of the field. The area of the regions has been chosen to vary in the range $10^{3} \div 10^{4} \operatorname{arcsec}^{2}$. The excess with respect to the background, if any, is never significant at $>3 \sigma$ confidence level. Counts were converted to fluxes assuming a Raymond-Smith model with $T=1 \mathrm{keV}$, and a solar abundances ratio of 0.4 . The $3 \sigma$ upper limit on the flux is then $2.7 \times 10^{-14} \mathrm{erg} \mathrm{cm}^{-2} \mathrm{~s}^{-1} \operatorname{arcmin}^{-2}$. Thus we can conclude that any diffuse emission is too faint to be detected.

\subsection{Sky coverage}

"Sky coverage" defines the area of the sky sensitive to a given flux limit, as a function of flux. Due to vignetting effects and to the increase of the size of the PSF in the outer regions of the detector, the center of the ACIS-I field of view is the most sensitive: i.e. for a given exposure time, the central part of the

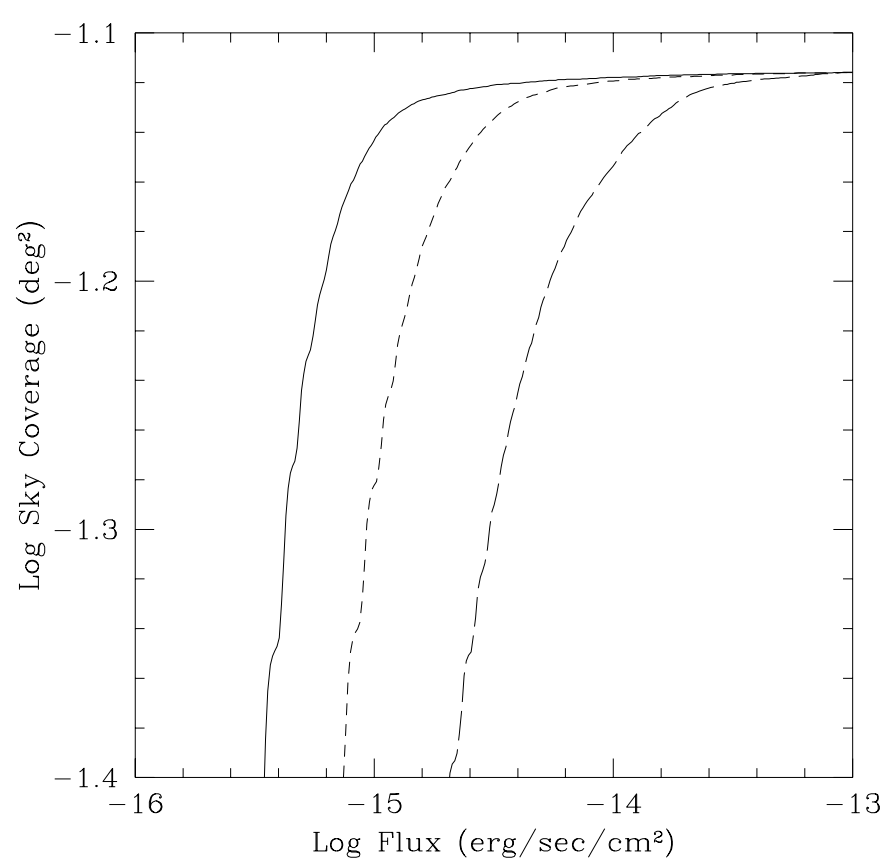

Fig. 3. The sky coverage (area covered vs. flux limit) for the $0.5-2 \mathrm{keV}$ band (solid line), for $2-10 \mathrm{keV}$ band (long dashed line) and for the 0.5-10 keV band (short dashed line).

detector is able to detect fainter sources than the outer regions. Thus, one must take into account this effect while computing the $\log N-\log S$, that is, the number of sources whose flux exceeds a given flux limit.

As a preliminary step to evaluating the sky coverage, we computed background maps for the two energy bands using the Ximage package. We did this by subtracting the central part of the field (the same $\sim 2^{\prime} \times 2^{\prime}$ region centered on the $3 \mathrm{C} 295$ cluster excluded from the source detection) and excluding all the sources detected in the images. In order to calculate the sky coverage, these maps and the exposure maps were rebinned into $128 \times 128$ matrices of $\sim 10$ arcsecs per pixel.

We also studied the increasing apparent size of the detected sources with the off-axis angle (i.e., the distance of the source from the aim point); both quantities are supplied by the detection algorithm. We used the rebinned background maps to calculate the background for all the points in the field in different size regions according to the relation between apparent size and off-axis. The local background level was used to calculate the minimum number of counts needed to exceed the noise in the case of Poisson statistics, to exceed the probability threshold of $2 \times 10^{-5}$. This value assures a number of spurious sources per field $<1$ in all energy bands (see previous subsection). The minimum counts were divided by the exposure map and thus converted to minimum detectable fluxes. The sky coverage at a given flux $f$ is simply the sum of all the regions of the detector whose minimum detectable flux is lower than $f$, and can be easily converted into deg ${ }^{2}$. The sky coverage computed with such a method for the three energy bands is shown in Fig. 3. 


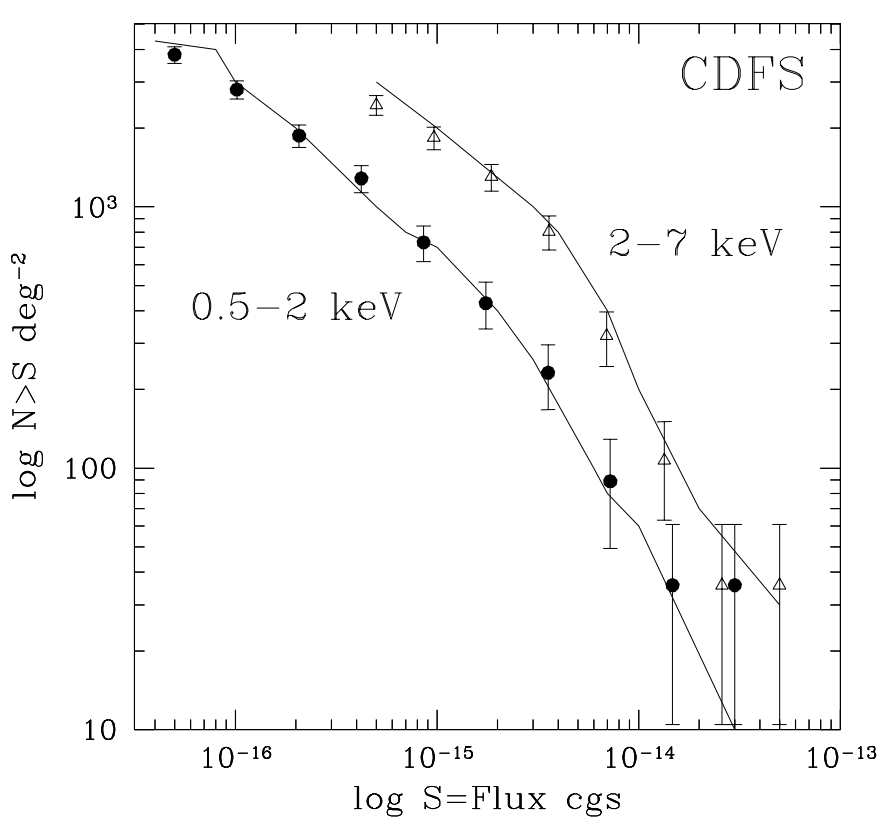

Fig. 4. The Chandra Deep Field South $\log N-\log S$ in the $0.5-2 \mathrm{keV}$ band (filled circles) and in the $2-10 \mathrm{keV}$ band (open triangles) evaluated in this paper. Errors represent $1 \sigma$ confidence limit. Solid lines represent the CDFS $\log N-\log S$ from Rosati et al. (2002).

\section{Results}

\section{1. $\log N-\log S$}

\subsubsection{Checking the analysis method}

To check the validity of our analysis method, we computed the $\log N-\log S$ of the CDFS and we compared this with previous computations (Rosati et al. 2002); the agreement is quite good (see Fig. 4). Then we computed the $\log N-\log S$ for $3 C 295$ in the three energy bands weighting the number of sources at a given flux with the sky coverage at the same flux; in Fig. 5 is shown the $\log N-\log S$ for the $0.5-2$ and 2-7 keV band. The CDFS $\log N-\log S$ extends to lower fluxes than the 3C 295 one, because the exposure time is about 10 times longer, and the error are similar in the region of flux overlap for the two field. There is excellent agreement between the number counts for the two fields.

\subsubsection{Chip-by-chip $\log N-\log S$}

While the average 3C $295 \log N-\log S$ appears normal, our goal is to search for inhomogenities within the ACIS-I field. The most natural way to do it is to derive the $\log N-\log S$ for each $8^{\prime} \times 8^{\prime}$ ACIS-I chip, separately. The $\log N-\log S$ for each ACIS-I chip, in each of the three bands, is shown in Fig. 6 . The $\log N-\log S$ in the upper left quadrant is significantly higher than that of the lower right chip, up to $2 \times 10^{-15} \mathrm{erg} \mathrm{cm}^{-2} \mathrm{~s}^{-1}$ in the $0.5-2 \mathrm{keV}$ band, up to $6 \times 10^{-15} \mathrm{erg} \mathrm{cm}^{-2} \mathrm{~s}^{-1}$ in the $2-10 \mathrm{keV}$ band, and up to $10^{-14} \mathrm{erg} \mathrm{cm}^{-2} \mathrm{~s}^{-1}$ in the $0.5-10 \mathrm{keV}$ one.

To be more quantitative, we fitted a power law to the four ACIS-I $\log N-\log S$ in each band, in the $3 \times$ $10^{-16}-2 \times 10^{-14} \mathrm{erg} \mathrm{cm}^{-2} \mathrm{~s}^{-1}$ flux range for the soft band,

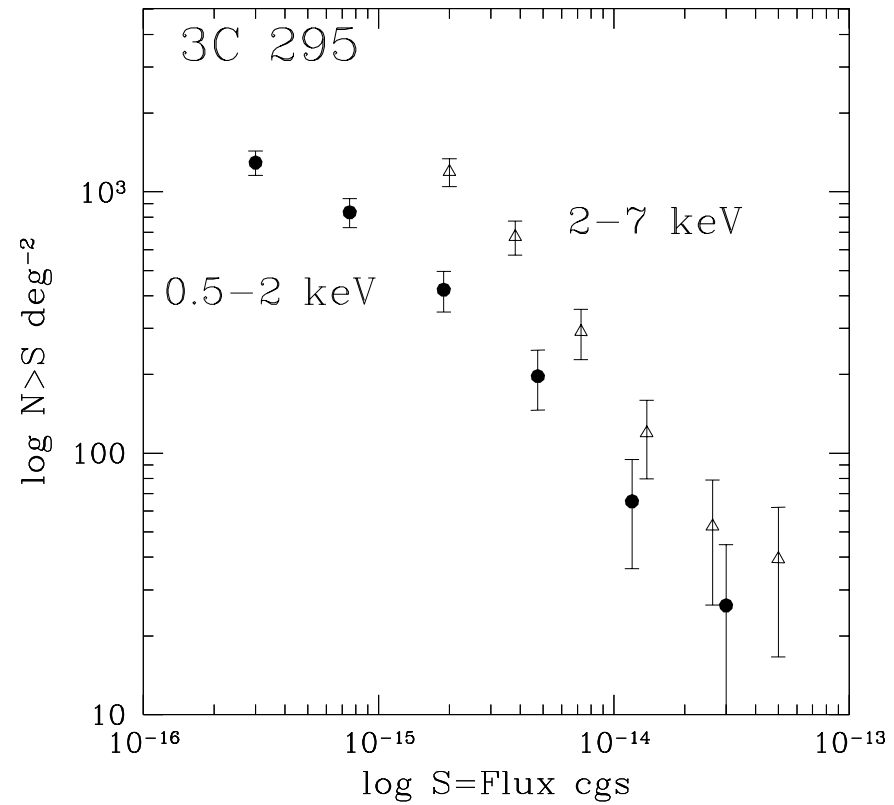

Fig. 5. The 3C $295 \log N-\log S$ in the $0.5-2 \mathrm{keV}$ band (filled circles) and in the $2-10 \mathrm{keV}$ band (open triangles) evaluated in this paper. Errors represent $1 \sigma$ confidence limit.

$3 \times 10^{-15}-2 \times 10^{-14} \mathrm{erg} \mathrm{cm}^{-2} \mathrm{~s}^{-1}$ for the hard band, and $6 \times 10^{-16}-2 \times 10^{-14} \mathrm{erg} \mathrm{cm}^{-2} \mathrm{~s}^{-1}$ for the $0.5-10 \mathrm{keV}$ band. Table 2 shows the results of the $\log N-\log S$ fits for the two fields. In Fig. 7 we plot the slope of the $\log N-\log S$ power law versus the normalization at $5 \times 10^{-16} \mathrm{erg} \mathrm{cm}^{-2} \mathrm{~s}^{-1}$ for the soft band, at $5 \times 10^{-15}$ erg $\mathrm{cm}^{-2} \mathrm{~s}^{-1}$ for the hard band and at $10^{-15} \mathrm{erg} \mathrm{cm}^{-2} \mathrm{~s}^{-1}$ for the total band; error bars represent the $90 \%$ confidence limit. Although the slopes of the four $\log N-\log S$ are all compatible within the errors, the normalization of the NE $\log N-\log S$ chip is clearly higher than that of the three other chips for all three bands. The best fit for the CDFS is shown with dashed lines and appear to be consistent with the latter chips, both for slope and normalization. The NE chip differs in normalization from the SW one by $3.2 \sigma$ in the soft band, by $3.3 \sigma$ in the hard one and by $4.0 \sigma$ in the total band. We note that in the total band, the significance of the discrepancy between the NE and SE chip is higher. This is due to the higher statistics that can be achieved using the whole energy range of the observation.

In the next subsections we will study in more detail the distribution of the sources, in particular how much it differs from the uniform distribution, and its angular correlation function.

\subsection{Two-dimensional Kolmogorov-Smirnov test}

In this subsection we apply a two-dimensional Kolmogorov-Smirnov (KS) test to check whether the distribution of the sources is uniform or not. The 1-D KS test is a simple and powerful tool to test whether two data sets are drawn from the same distribution, or whether a data set is compatible with a given distribution, provided that these data sets and distributions are functions of only one variable. The KS test makes use of the parameter $D$, which represents the maximum value of the absolute difference between the 

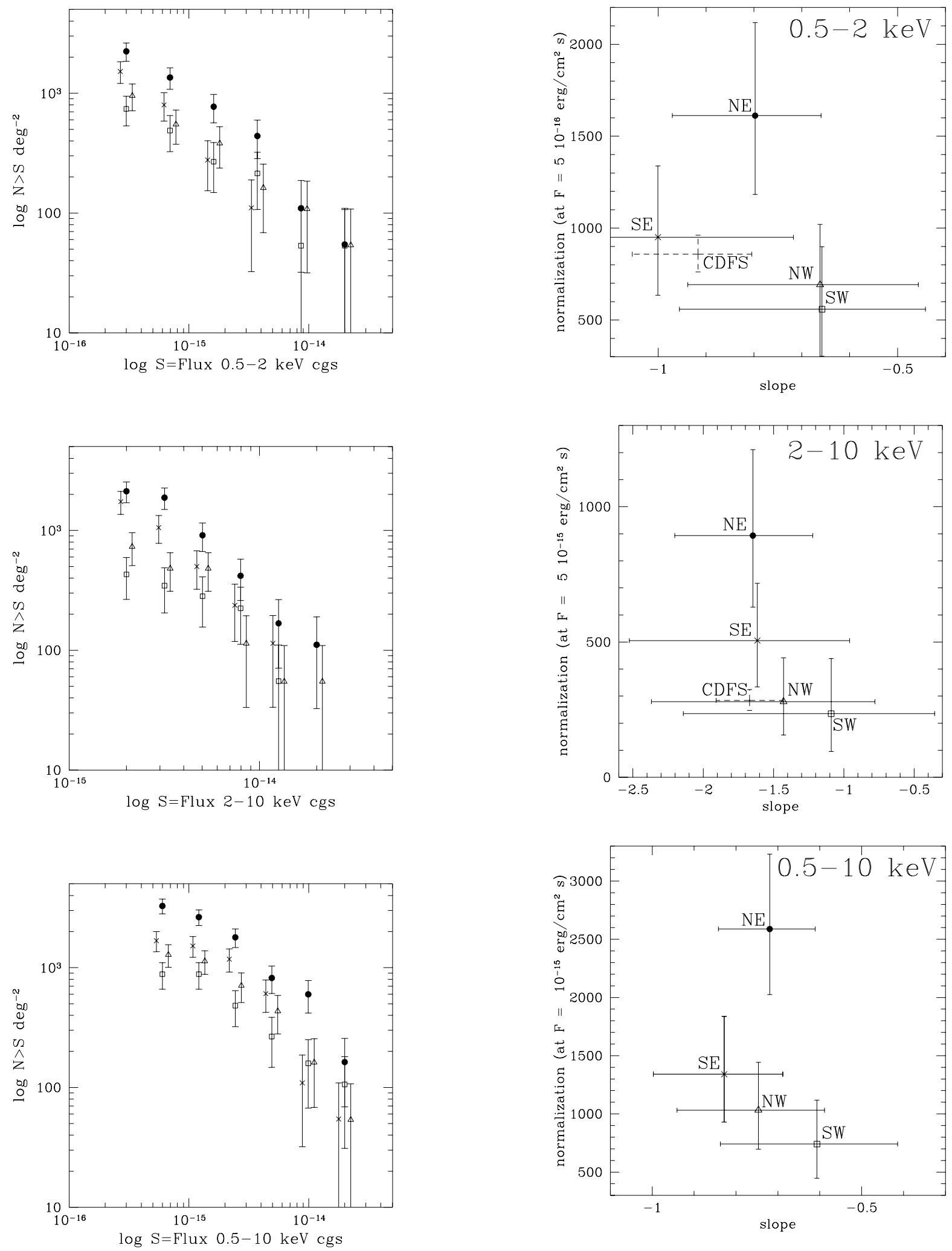

Fig. 6. The mean (whole field) $3 \mathrm{C} 295 \log N-\log S$ in the $0.5-2 \mathrm{keV}$ band (top panel), in the $2-10 \mathrm{KeV}$ band (central panel) and in the 0.5-10 keV band (bottom panel), calculated for each ACIS-I chip separately. Filled circles represent counts for the NE chip, open triangles for the NW, open squares for the SW and crosses for the SE. For clarity, the points for the SE chip and the NW have been shifted slightly to the left and to the right, respectively. Errors represent $1 \sigma$ confidence limit. in the soft band (top panel), in the hard band (central panel) and for the whole band (bottom panel). $x$ axis plots the slope of the power law, while on the $y$ axis is plotted the normalization at $5 \times$ $10^{-16} \mathrm{erg} \mathrm{cm}^{-2} \mathrm{~s}^{-1}$ (soft band), at $5 \times 10^{-15} \mathrm{erg} \mathrm{cm}^{-2} \mathrm{~s}^{-1}$ (hard band) and at $10^{-16} \mathrm{erg} \mathrm{cm}^{-2} \mathrm{~s}^{-1}$ (whole band). Filled circles represent the $\mathrm{NE}$ chip, open triangles the NW, open squares the SW and crosses the SE. Dashed lines represent the fit for the CDFS. Errors are the $90 \%$ confidence limit. 
Table 2. Results of the four 3C 295 chips and CDFS $\log N-\log S$ fit.

\begin{tabular}{l|ccc|ccc|ccc}
\hline \hline & \multicolumn{3}{|c|}{$0.5-2 \mathrm{keV}$} & \multicolumn{3}{c|}{$2-10 \mathrm{keV}$} & \multicolumn{3}{c}{$0.5-10 \mathrm{keV}$} \\
\hline & Sources & Slope & Normaliz. & Sources & Slope & Normaliz. & Sources & Slope & Normaliz. \\
\hline 3C 295 & 89 & $-0.75_{-0.1}^{+0.09}$ & $1000_{-100}^{+100}$ & 71 & $-1.3_{-0.2}^{+0.2}$ & $390_{-50}^{+60}$ & 121 & $-0.9_{-0.2}^{+0.1}$ & $1300_{-200}^{+200}$ \\
$\mathrm{NE}$ & 36 & $-0.8_{-0.2}^{+0.1}$ & $1600_{-400}^{+600}$ & 29 & $-1.6_{-0.6}^{+0.4}$ & $900_{-300}^{+300}$ & 53 & $-0.7_{-0.1}^{+0.1}$ & $2600_{-600}^{+600}$ \\
$\mathrm{NW}$ & 18 & $-0.7_{-0.3}^{+0.2}$ & $700_{-400}^{+300}$ & 13 & $-1.4_{-0.9}^{+0.7}$ & $300_{-100}^{+200}$ & 23 & $-0.7_{-0.2}^{+0.2}$ & $1000_{-300}^{+300}$ \\
$\mathrm{SW}$ & 12 & $-0.7_{-0.3}^{+0.2}$ & $600_{-300}^{+300}$ & 7 & $-1.1_{-1.0}^{+0.7}$ & $200_{-100}^{+200}$ & 17 & $-0.6_{-0.2}^{+0.2}$ & $700_{-300}^{+400}$ \\
$\mathrm{SE}$ & 23 & $-1.0_{-0.4}^{+0.3}$ & $950_{-300}^{+400}$ & 22 & $-1.6_{-0.9}^{+0.7}$ & $500_{-200}^{+200}$ & 28 & $-0.8_{-0.2}^{+0.1}$ & $1300_{-300}^{+500}$ \\
$\mathrm{CDFS}$ & 202 & $-0.9_{-0.1}^{+0.1}$ & $900_{-100}^{+100}$ & 151 & $-1.7_{-0.2}^{+0.2}$ & $280_{-40}^{+40}$ & - & - & - \\
\hline
\end{tabular}

cumulative distributions drawn from the two data sets, or from the data set and the given distribution. Once $D$ is known, the probability that a larger value of $D$ can be observed if the two distributions are identical is given by:

$P(D>$ observed $)=2 \sum_{i=1}^{\infty}(-1)^{i-1} \mathrm{e}^{-2 i^{2} \lambda^{2}}$,

where $\lambda$ is a simple function of $D$.

The generalization of the KS test to a two-dimensional distribution is due to Peacock (1983) and Fasano \& Franceschini (1987). Any point of a data set is now defined by a pair of coordinates, which in turn define a plane. Thus, given a point of the data set, one can compute how many elements (of the same data set or of another one) occupy the four quadrants defined by such a point, or how many points are expected in the four quadrants according to the given distribution. The parameter $D$ can now be defined as the maximum value of the absolute difference between the number of elements of the two data set (or of the data set and the distribution) in the quadrants, as the reference point changes within the data set. Fasano \& Franceschini (1987) demonstrated that Eq. (1) holds even for two-dimensional distributions, provided that

$\lambda=\frac{\sqrt{N} D}{1+\sqrt{1-r^{2}}(0.25-0.75 / \sqrt{N})} ;$

here $N$ is the number of elements of the data set (or $N=$ $N_{1} N_{2} /\left(N_{1}+N_{2}\right)$ if two data sets are involved) and $r$ is the correlation coefficient of the two variables of the data set (or the average between the two correlation coefficients in the two data set case).

We have used Eqs. (1) and (2) to test the hypothesis that the distribution of the sources in the 3C 295 field is uniform. Since the sources are not uniformly distributed due to the sensitivity of the instrument being higher in the middle of the detector (see Sect. 2.2), low flux sources can only be found near the centre of the four ACIS-I chips, so we imposed a higher minimum flux. We specified this minimum flux as the one which could have been detected at least in $75 \%$ of the detector area. We note that this effect is not very strong in the 3C 295 field, because the central part of the detector was excluded due to the presence of the cluster. We found the probability that the distribution of the 3C 295 sources being uniform is very low: $3 \%, \sim 4 \%$ and $\sim 0.6 \%$ in the $0.5-2,2-7$ and $0.5-7 \mathrm{keV}$ bands, respectively (see also Table 3 ).

\subsection{Angular correlation function}

Although the $\log N-\log S$ and the 2-D KS test show that the sources in the 3C 295 field are not uniformily distributed, they do not give angular information on the spatial distribution of the sources. The conventional way to determine the spatial correlation of sources in a field is to use the "two point angular correlation function" (ACF; Peebles 1980; Peacock 1999). This function measures the strength of the spatial correlation, but does not say anything about where the sources are clustered. This function is given by the following relation:

$w(\theta)=\frac{D D}{R R}-1$,

where $D D$ is the ratio of pairs of sources in the real data, which fall in the separation angle $(\theta, \theta+\mathrm{d} \theta)$ and the $R R$ is the number of those pairs expected when the spatial distribution of sources is random. To compute the ACFs, we proceeded as follows: we computed the angular separation for the pairs in our data set; we generated a random uniform distribution of 1000 sources in the same area and we computed their angular separation; we evaluated the ACFs using Eq. (3) and rescaling $R R$ to the number of pairs of the real field. We made the same flux cutoff to take into account the higher sensitivity of the detector in its central region (see previous subsection). Figure 8 shows the ACFs for the 3C 295 field.

The errors in Fig. 8 were estimated using two different methods. The first one (solid lines) is simply the Poisson statistics, the error is defined as the inverse of the square root of the real pair number (Peebles 1980) and is show in Fig. 8 with solid lines. The second one (dashed lines) is more accurate and is called the bootstrap resampling method (see Barrow et al. 1984, and references therein). Briefly, we generated 100 simulated fields from the real one, by randomly selecting within the sources of the field a number of sources equal to the data set size. This produces simulated fields with a number of elements equal to that of the real field, but with some sources counted more than once and others not considered at all. The bootstrap method consists of recalculating the ACF using such simulated fields and in calculating the error as the standard deviation of the $100 \mathrm{ACFs}$ obtained in this way. The bootstrap errors (which 

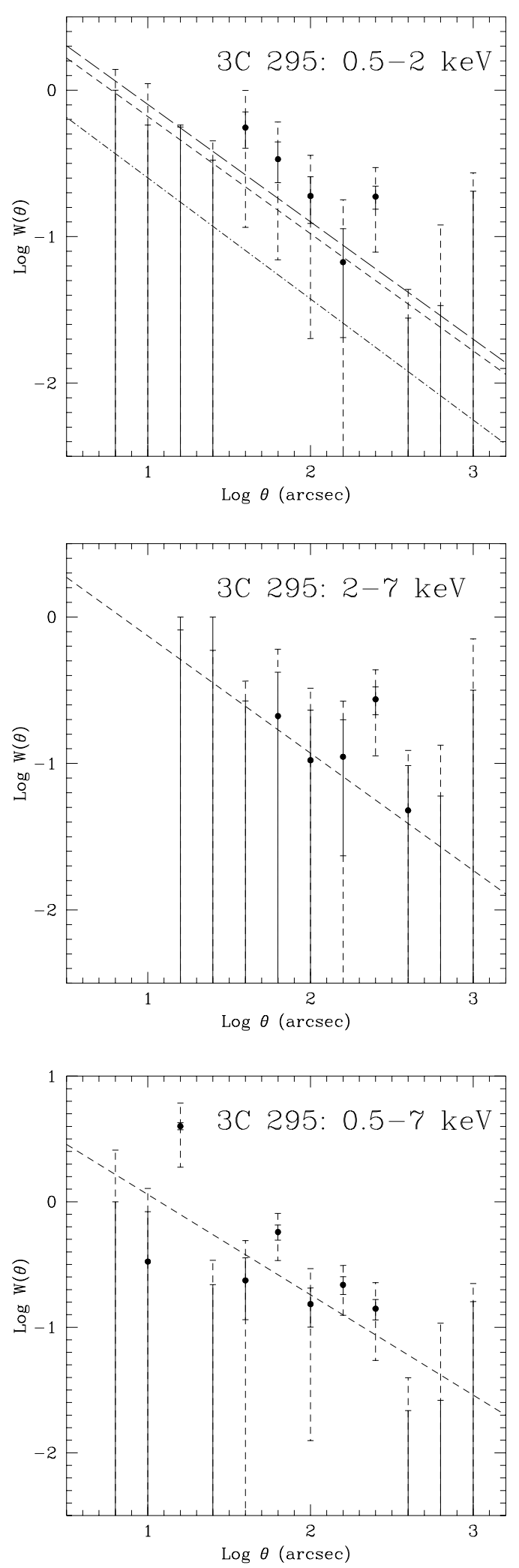

Fig. 8. The 3C 295 angular correlation function in the $0.5-2 \mathrm{keV}$ band (top panel), in the 2-7 keV (central panel) and in the $0.5-7 \mathrm{keV}$ band (bottom panel). Solid error bars are Poisson; dashed error bars are bootstrap. Short-dashed lines represent our fit to the data assuming bootstrap error bars. The long dashed line represents the best fit found by Vikhlinin \& Forman (1995) for ROSAT sources; the dot-dashed line is the simulated ACF calculated by the same authors in order to correct the effect of the amplification bias. probably overestimate the true error) exceed the Poisson ones. This is because the Poisson errors depend on the number of pairs at each scale, while the bootstrap ones also depend on how many pairs are produced by each single source. In more detail, let us suppose that a large number of sources are distributed along a circumference, and one is placed at its centre; obviously, a strong angular correlation will be found at scales equal to the circle's radius. Poisson errors will be strongly reduced as the number of sources along the circumference increases, ignoring that a single source (namely, the central one) strongly boosts the correlation; bootstrap errors take this effect into account by randomly excluding or counting more than once the central source, when recomputing the ACF.

Figure 8 (long dashed line) also shows the best fit for the angular correlation function found by Vikhlinin \& Forman (1995) for an extensive set of deep ROSAT observations covering $40 \mathrm{deg}^{2}$ of sky. Their power law model $w(\theta)=\left(\theta / \theta_{0}\right)^{(1-\gamma)}$ yields the best fit parameters $\theta_{0}=10 \pm 8$ arcsec and $\gamma=1.7 \pm 0.3$. However they found that the correlation angle $\theta_{0}$ was smaller than the $F W H M$ of the ROSAT PSPC PSF, which is $\sim 25$ arcsec on-axis, which leads to an amplification bias. Simulations to correct for this gave the ACF show in Fig. 8 with a dot-dashed line, which has a normalization 2.85 times smaller (Vikhlinin \& Forman 1995). This means that the uncorrected Vikhlinin \& Forman (1995) ACF (long dashed line) represents a strong upper limit to the ROSAT ACF. Since the ROSAT PSPC energy band is $0.1-2 \mathrm{keV}$ we cannot compare the Vikhlinin \& Forman (1995) ACF with our ACFs in the hard (2-7 keV) and whole (0.5-7 keV) bands.

Vikhlinin \& Forman (1995) sample could only produce the ACF down to a $230 \operatorname{arcsec}$ scale $(\log \theta=1.5)$.

For the 3C 295 field in the $0.5-2 \mathrm{keV}$ band, we found a signal above the upper limit of the Vikhlinin \& Forman (1995) fit on scales $\sim 60-240$ arcsec, if we use the Poissonian error bars; however using the bootstrap errors reduces this scale to only $\sim 240$ arcsec. A similar effect is present in the $2-7 \mathrm{keV}$ band, where a clear signal of correlation emerges on scales of $\sim 240$ arcsec assuming both Poisson and bootstrap statistics. A stronger and wider signal is present in the $0.5-7 \mathrm{keV}$ band, on scales ranging from $\sim 15 \operatorname{arcsec}$ to $\sim 240 \mathrm{arcsec}$. This reflects the clustering of the sources clearly visible in the upper left corner of Fig. 1 (on linear scales of the order of half a chip length, $\sim 240$ arcsec). The improved statistics in the $0.5-7 \mathrm{keV}$ band, due to the higher number of sources detected, gives rise to a stronger signal.

The short-dashed lines in Fig. 8 represent the fit of $w(\theta)=$ $\left(\theta / \theta_{0}\right)^{1-\gamma}$ to our data, assuming the bootstrap error bars; $\gamma=$ 1.8 was fixed as in Vikhlinin \& Forman (1995). In the soft band we found $\theta_{0}=6.0_{-3.7}^{+4.4}$ arcsec, in the hard band $\theta_{0}=$ $6.9_{-5.1}^{+6.9}$ arcsec, and in the broad band $\theta_{0}=8.5_{-4.5}^{+6.5} \operatorname{arcsec}(90 \%$ confidence limit). These values are consistent within their relatively large error to the Vikhlinin \& Forman (1995) fit for $\theta_{0}$ without correcting for the amplification bias $\left(\theta_{0} \sim 10 \operatorname{arcsec}\right)$. If we assume the Poisson error bars, the value of $\theta_{0}$ rises and the errors become smaller in all energy bands.

Finally, we checked the dependance of the ACF on the flux limit. We computed the ACF in the broad band for three different flux limits (i.e., 1 times, 2 times and 3 times the flux limit 
given in Table 3 , footnote $a$ ) and we found that $\theta_{0}$ vary less than $25 \%$, while its error increases with the flux limit.

\subsubsection{CDFS}

We applied the KS test and evaluated the ACF for the CDFS to check whether this field is different from 3C 295.

Table 3 shows the results of the two dimensional KS test. We can see that CDFS is compatible with a uniform distribution with a higher probability $(\sim 15 \%)$ than 3c 295 ( $\sim$ a few \%). We note that the KS test result depends on the minimum flux chosen; nevertheless, KS for CDFS gives a higher probability of a uniform distribution than 3C 295 even if we use for the two observations the same flux limit. The first and the third column of Table 3 report the KS results for the flux limit described in this subsection; the second column shows the CDFS results for a flux limit equal to that of $3 \mathrm{C} 295$.

In Fig. 9 we show the ACF for the CDFS field; in both the energy bands we found no evidence of a strong signal such as that shown by the 3C 295 field. Giacconi et al. (2001) evaluated the CDFS ACF for the first $100 \mathrm{ks}$ observation of the CDFS; so, we checked our algorithm by calculating the ACF for the same data and found, reassuringly, agreement within $1 \sigma$.

\subsubsection{ACF in 8 selected Chandra fields}

To check whether the strong clustering of the 3C 295 field is peculiar, the comparison with the CDFS is not enough, and a larger sample of Chandra ACIS-I fields of similar exposure time has to be analyzed. For this reason, we browsed the Chandra data archive and matched all the ACIS-I observations belonging to the classes "AGNs" and "Surveys", with an exposure time of at least $50 \mathrm{ks}$. We used the detection algorithm PWDetect on these fields to detect sources in the three energy bands. In Table 4 we show the 8 fields selected for this analysis, their exposure times and the sources detected in the three bands.

We computed the ACF in the $0.5-7 \mathrm{keV}$ band using a flux cut-off such that the minimum flux could have been detected at least in $75 \%$ of the detector area, as we did for 3C 295. The average ACF of this sample is shown in Fig. 10. Although a signal is still present at scales $>100$ arcsec, it is clearly weaker than that of 3C 295. We fitted these data with a power law of fixed slope $1-\gamma, \gamma=1.8$ as we did for the 3C $295 \mathrm{ACF}$, and we found the correlation angle $\theta_{0}=1.2_{-1.0}^{+1.3} \operatorname{arcsec}(90 \%$ confidence limit). This is lower than that of $3 C 295$ by a factor of $\gtrsim 2$ at a confidence level of $2.5 \sigma$.

\section{Discussion}

A 92 ks Chandra observation of the source field around the $z=0.46$ 3C 295 cluster shows an excess of sources visible in the NE corner of the Chandra observation (Fig. 1). This is clear in the density contour plot of Fig. 11. This figure shows that the denser region of the putative filament has a source density more than 5 times higher than the average source density of the field $\left(\sim 0.5\right.$ sources per $\left.\operatorname{arcmin}^{2}\right)$. A chip by chip $\log N-\log S$ analysis demonstrates that the NE chip has a $4.0 \sigma$ excess of sources
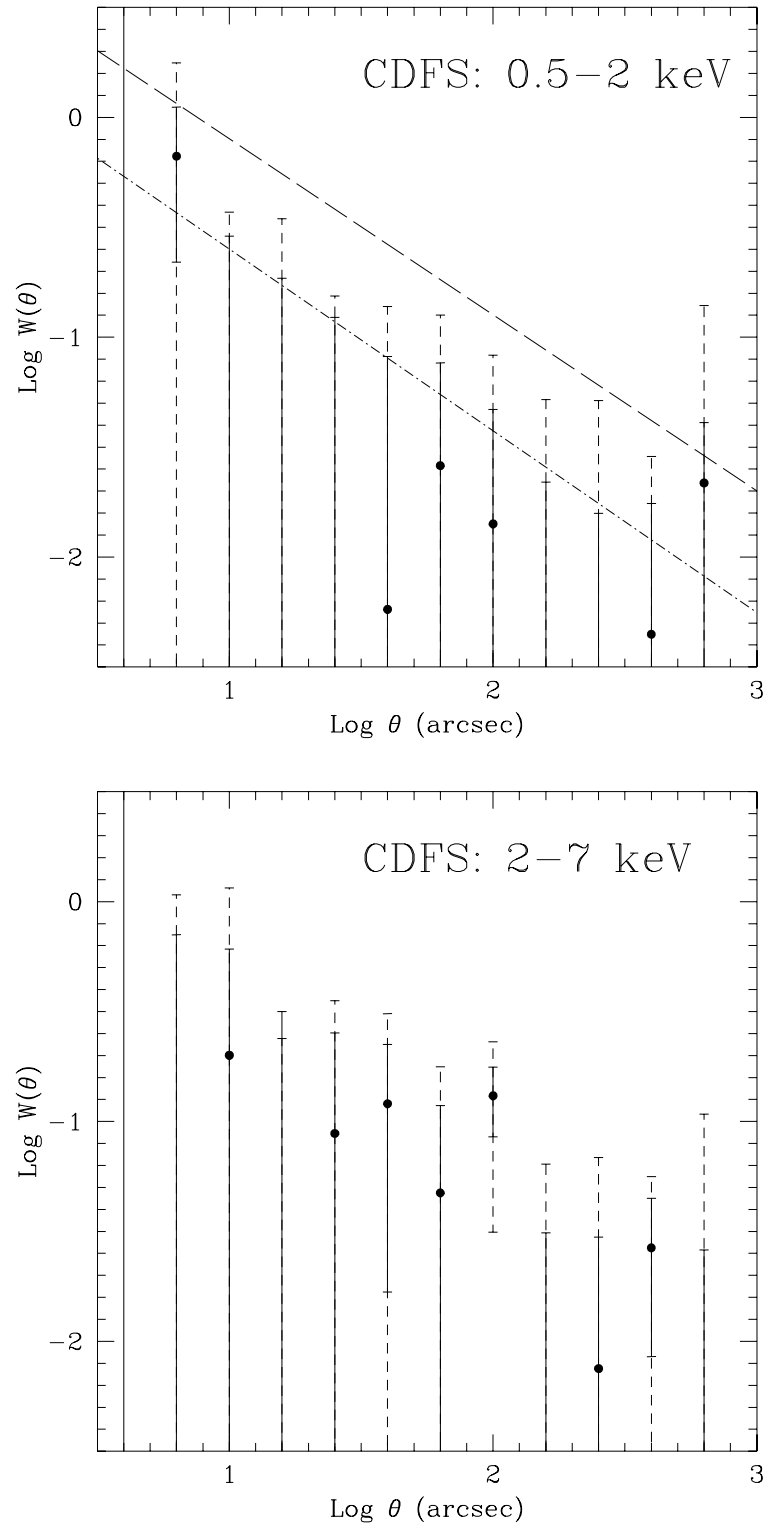

Fig. 9. The CDFS angular correlation function in the $0.5-2 \mathrm{keV}$ band (top panel) and in the $2-7 \mathrm{keV}$ (bottom panel). The dashed line line represents the best fit found by Vikhlinin \& Forman (1995) for ROSAT sources; the dot-dashed line is the simulated ACF calculated by the same authors to correct the effect of the amplification bias. Solid error bars are Poisson; dashed error bars are bootstrap.

over the SW chip in the total $(0.5-7 \mathrm{keV})$ Chanda band. (In the standard soft $(0.5-2 \mathrm{keV})$ band the excess is $3.2 \sigma$, and in the standard hard (2-7 keV) band the excess is $3.3 \sigma$.) This result confirms the basic result of Cappi et al. (2001) and extends it to deeper fluxes, larger fields-of-view and the $2-10 \mathrm{keV}$ band.

The asymmetric distribution of the 3C 295 field is confirmed by two analyses: (1) the two dimensional KolmogorovSmirnov test, which shows that the probability that the sources are uniformly distributed is $0.632 \%$ in the total Chandra band (3.09\% for the soft band and $3.84 \%$ for the hard band); (2) the two point angular correlation function (ACF) strongly indicates a positive correlation for scales of $0.5-5$ arcmin. This strong correlation has not been found in a sample of eight 


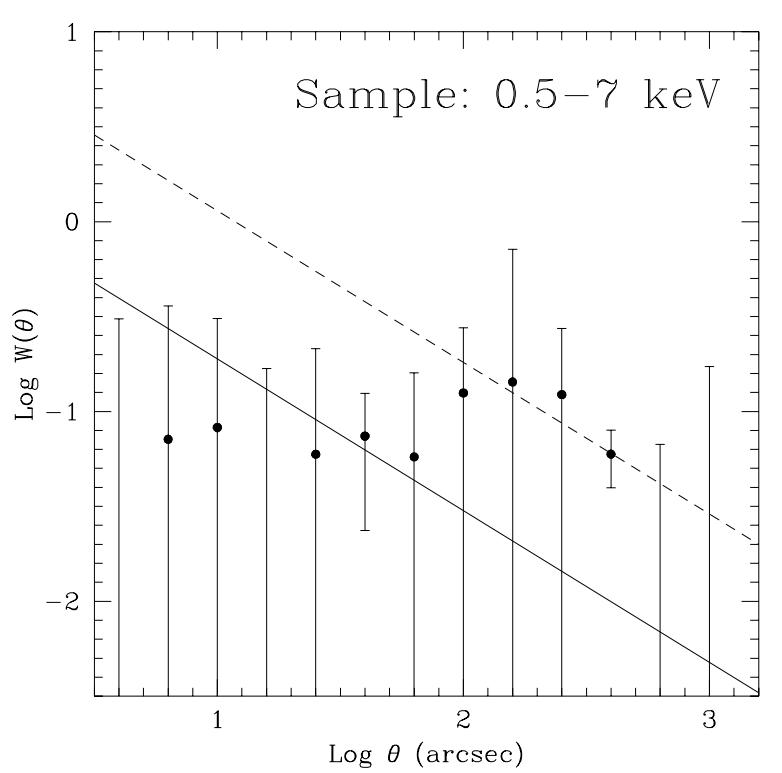

Fig. 10. The average angular correlation function in the $0.5-7 \mathrm{keV}$ band for the sample of fields reported in Table 4; Error bars are bootstraps. The solid line represents the best fit for these data; the dashed line is the fit for the ACF of 3C 295 (see also Fig. 8, bottom panel).

Table 3. KS test for CDFS and 3C 295 fields.

\begin{tabular}{lccc}
\hline \hline & $3 \mathrm{C} 295^{a}$ & $\mathrm{CDFS}^{a}$ & $\mathrm{CDFS}^{b}$ \\
\hline $0.5-2 \mathrm{keV}$ & $3.09 \times 10^{-2}$ & 0.2 & 0.13 \\
$2-7 \mathrm{keV}$ & $3.84 \times 10^{-2}$ & 0.5 & 0.17 \\
$0.5-7 \mathrm{keV}$ & $6.32 \times 10^{-3}$ & - & - \\
\hline
\end{tabular}

${ }^{a}$ Lux limits: $0.5-2 \mathrm{keV}, F_{\mathrm{X}}>4.5 \times 10^{-16} \mathrm{erg} \mathrm{s}^{-1} \mathrm{~cm}^{-2} ; 2-7 \mathrm{keV}, F_{\mathrm{X}}>$ $3.5 \times 10^{-15} \mathrm{erg} \mathrm{s}^{-1} \mathrm{~cm}^{-2} ; 0.5-7 \mathrm{keV}, F_{\mathrm{X}}>7.8 \times 10^{-16} \mathrm{erg} \mathrm{s}^{-1} \mathrm{~cm}^{-2}$.

${ }^{b}$ Flux limits: $0.5-2 \mathrm{keV}, F_{\mathrm{X}}>6.0 \times 10^{-17} \mathrm{erg} \mathrm{s}^{-1} \mathrm{~cm}^{-2} 2-7 \mathrm{keV}$, $F_{\mathrm{X}}>5.0 \times 10^{-16} \mathrm{erg} \mathrm{s}^{-1} \mathrm{~cm}^{-2}$.

ACIS-I fields with a similar exposure time, for which the correlation angle $\theta_{0}$ is smaller by a factor of $\gtrsim 2$ than that of 3C 295 at the $2.5 \sigma$ confidence level, suggesting that the 3C 295 overdensity of sources in the NE chip is truly peculiar. An intriguing explanation, to be confirmed when the redshifts of the sources are measured, is that the overdensity of sources is actually related to a cosmic filament of the LSS.

Cappi et al. (2001) discussed four possible causes for this "surplus" of sources: (1) gravitationally lensed very faint sources; (2) rapid evolution of cluster AGN or starburst galaxies; (3) cosmic variance of background sources; (4) LSS associated with the clusters. Since the surplus sources are not symmetrically placed around the cluster, our results rule out an enhanced AGN population in the 3C 295 cluster itself, and lensing by the cluster potential. Since $N$-body and hydrodynamical simulations and galaxy surveys (e.g $2 \mathrm{dF}$ and Sloan) lead us to expect that clusters of galaxies lie at the nexus of several filaments, we believe that this excess is likely to represent a filament of the LSS of the Universe converging onto the 3C 295 cluster.

The redshifts of the sources making up the excess are not yet known. However, if we assume that the sources are

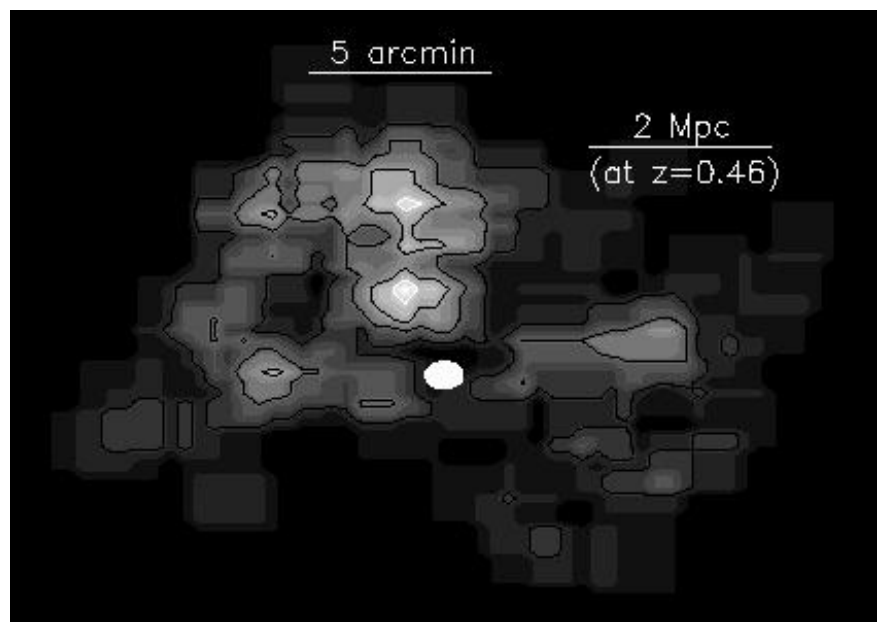

Fig. 11. The density profile of the 3C 295 field, computed for the whole $0.5-7 \mathrm{keV}$ band. The linear smoothing factor is 1.5 arcmin, and the four contour levels indicate source densities of 1.3, 1.8, 2.2 and 2.7 sources per $\operatorname{arcmin}^{2}$. The clustering of sources in the NE corner is clearly visible; the bright central spot is the 3C 295 cluster.

associated with the 3C 295 cluster, then we can use the cluster redshift to estimate some of their properties.

Adopting a concordance cosmology $\left(H_{0}=65 \mathrm{~km} \mathrm{~s}^{-1} \mathrm{Mpc}\right.$, $\Omega_{M}=0.3$ and $\Omega_{\Lambda}=0.7$, Spergel et al. 2003) we obtain luminosities in the range $7.5 \times 10^{41} \div 1.1 \times 10^{44} \mathrm{erg} \mathrm{s}^{-1}$ (median $\left.=3.1 \times 10^{42} \mathrm{erg} \mathrm{s}^{-1}\right)$ for the total Chandra band. $(2.8 \times$ $10^{41} \div 3.8 \times 10^{43} \mathrm{erg} \mathrm{s}^{-1}\left(\right.$ median $\left.=1.1 \times 10^{42} \mathrm{erg} \mathrm{s}^{-1}\right)$ for the soft band; $1.5 \times 10^{42} \div 5.0 \times 10^{43} \mathrm{erg} \mathrm{s}^{-1}\left(\right.$ median $=4.5 \times 10^{42} \mathrm{erg} \mathrm{s}^{-1}$ ) for the hard band.) These are moderate, Seyfert galaxy luminosities, extending down to the range of luminous starburst galaxies at the faint end of the soft band.

An association with 3C 295 also defines a spatial scale of $2 \mathrm{Mpc}(5 \mathrm{arcmin})$ for the adopted cosmology. For an, admittedly unlikely, spherical region of volume of $\sim 4 \mathrm{Mpc}^{3}$ containing the excess sources, the implied space density of the excess sources (i.e. subtracting the CDF-S defined $\log N-\log S$ contribution) is $0.9 \mathrm{Mpc}^{-3}(0.5-2 \mathrm{keV})$ and $0.8 \mathrm{Mpc}^{-3}(2-10 \mathrm{keV})$. This is well above the normal maximum AGN space density at $z=0.5\left(\sim 10^{-4} \mathrm{Mpc}^{-3}\right.$, for luminosities down to $10^{42} \mathrm{erg} \mathrm{s}^{-1}$ in the $0.5-2 \mathrm{keV}$ band (Hasinger 2003) and $10^{43} \mathrm{erg} \mathrm{s}^{-1}$ in the $2-10 \mathrm{keV}$ band (Fiore et al. 2003). However, since the $\mathrm{X}$-ray source luminosities are as low as $\sim 2 \times 10^{41} \mathrm{erg} \mathrm{s}^{-1}$ (at the redshift of 3C 295), the little-studied lower end of the XLF is being probed, and contributions from starburst and even normal galaxies can be important.

The integral of the field galaxy luminosity function at $z=0.5$ (e.g., Poli et al. 2001) gives $\sim 0.13$ galaxies $\mathrm{Mpc}^{-3}$ for $M_{B}<-17$ (a resonable faint end optical luminosity, corresponding to our lower X-ray luminosities). If we assume that roughly one tenth of the galaxies are active $\mathrm{X}$-ray sources of $L_{\mathrm{X}}>3 \times 10^{41} \mathrm{erg} \mathrm{s}^{-1}$, then we would expect $\sim 0.013 \mathrm{X}$ ray sources $\mathrm{Mpc}^{-3}$. Since we count $\sim 0.9$ sources $\mathrm{Mpc}^{-3}$, this implies a galaxy overdensity of $\approx 70$, with of course a large (factor of 2-4) positive and negative uncertainty, because of the uncertainties in our space densities and assumptions. Still, this is intriguingly close to the expected galaxy overdensity of 
Table 4. Chandra fields chosen for the ACF analysis.

\begin{tabular}{l|c|c|c|c}
\hline \hline Field & Exposure time & \multicolumn{3}{|c}{ Number of sources } \\
\cline { 3 - 5 } & $(\mathrm{ks})$ & $0.5-2 \mathrm{keV}$ & $2-7 \mathrm{keV}$ & $0.5-7 \mathrm{keV}$ \\
\hline NGC55 & 60 & 108 & 75 & 132 \\
WHDF & 72 & 83 & 58 & 104 \\
ISO & 74 & 92 & 76 & 116 \\
GROTH-WEST.F & 85 & 84 & 63 & 103 \\
1156+295 & 75 & 145 & 50 & 150 \\
Elais:N1 & 74 & 101 & 80 & 127 \\
Elais:N2 & 74 & 85 & 60 & 102 \\
FIELD-142549+35 & 122 & 110 & 96 & 148 \\
\hline
\end{tabular}

filaments $\sim 10 \div 10^{2}$, and much smaller than the overdensities of clusters of galaxies $\left(\sim 10^{3} \div 10^{4}\right.$, the density contrast at the virial radius being $\sim 200$ ). Furthermore, in a filamentary structure the implied space density will depend strongly on the orientation of the filament to our line of sight.

We stress again that these arguments hold only under the assumption that the redshift of the excess sources is the same as that of the $3 \mathrm{C} 295$ cluster. To determine if this excess is associated with the 3C 295 cluster, we need to know the redshift of the sources via optical identifications.

For this reason, to study this candidate filament, we are pursuing new Chandra and optical observations to map the 3C 295 region and delimit the filament properties up to scales of $\sim 24$ arcmin (i.e. $\sim 6 \mathrm{Mpc}$ ) from the 3C 295 cluster. These studies are important because they may open up a new way to map high-density peaks of LSS at high redshifts with high efficiency.

Acknowledgements. We thank Dong-Woo Kim for careful reading of the manuscript and for useful comments. We are in debted to Salvo Sciortino, Giusi Micela and Francesco Damiani for providing us with the PWdetect algorithm and the relevant know-how.

\section{References}

Almaini, O., Scott, S. E., Dunlop, J. S., et al. 2003, MNRAS, 338, 303 Barrow, J. D., Bhavsar, S. P., \& Sonoda, D. H. 1984, MNRAS, 210, 19

Best, P. N., van Dokkum, P. G., Franx, M., \& Rottgering, H. J. A. 2002, MNRAS, 330, 17

Cappi, M., Mazzotta, P., Elvis, M., et al. 2001, ApJ, 548, 624

Daddi, E., Broadhurst, T., Zamorani, G., et al. 2001, A\&A, 376, 825

Damiani, F., Maggio, A., Micela, G., \& Sciortino, S. 1997a, ApJ, 483, 350

Damiani, F., Maggio, A., Micela, G., \& Sciortino, S. 1997b, ApJ, 483, 370

Davé, R., Spergel, D. N., Steinhardt, P. J., \& Wandelt, B. D. 2001, ApJ, 547, 574
Dickey, J. M., \& Lockman, F. J. 1990, ARA\&A, 28, 215

Dressler, A., \& Gunn, J. E. 1992, ApJS, 78, 1

Fasano, G., \& Franceschini, A. 1987, MNRAS, 225, 155

Fiore, et al. 2003, A\&A, submitted

Fiore, F., Nicastro, F., Savaglio, S., Stella, L., \& Vietri, M. 2000, ApJ, 544, L7

Garmire, G. P. 1997, A\&AS, 29, 283

Giacconi, R., Zirm, A., Wang, J., et al. 2002, ApJ, 139, 369

Giacconi, R., Rosati, P., Tozzi, P., et al. 2001, ApJ, 551, 624

Giavalisco, M., \& Dickinson, M. 2001, ApJ, 550, 177

Gilli, R., Cimatti, A., Daddi, E., et al. 2003, ApJ, accepted [arXiv: astro-ph/0304177]

Hasinger, 2003 [arXiv: astro-ph/0302574]

Hasinger, G., Burg, R., Giacconi, R., et al. 1998, A\&A, 329, 482

Henry, J. P., \& Henriksen, M. J. 1986, ApJ, 301, 689

Martini, P., Kelson, D. D., Mulchaey, J. S., \& Trager, S. C. 2002, ApJ, 576, L109

Mathur, S., Weinberg, D. H., \& Chen, X. 2003, ApJ, 582, 82

Molnar, S. M., Hughes, J. P., Donahue, M., \& Joy, M. 2002, ApJ, 573, L91

Nicastro, F., Zezas, A., Drake, J., et al. 2002, ApJ, 573, 157

Nicastro, F., Zezas, A., Elvis, M., et al. 2003, Nature, 421, 719

Peacock, J. A. 1983, MNRAS, 202, 615

Peacock, J. A. 1999, Cosmological physics (Cambridge: Cambridge University Press)

Peebles, P. J. E. 1980, The Large Scale Structure of the Universe (Princeton: Princeton University Press)

Pentericci, L., Kurk, J. D., Carilli, C. L., et al. 2002 [arXiv: astro-ph/0209392]

Poli, F., Giallongo, E., Fontana, A., Cristiani, S., \& D'Odorico, S. 2001, ApJ, 551, L45

Poli, F., Giallongo, E., Fontana, A., et al. 2003, ApJ, 593, L1

Rosati, P., Tozzi, P., Giacconi, R., et al. 2002, ApJ, 566, 667

Soltan, A. M., Freyberg, M. J., \& Hasinger, G. 2002, A\&A, 395, 475

Tozzi, P., Rosati, P., Nonino, M., et al. 2001, ApJ, 562, 42

Vikhlinin, A., \& Forman, W. 1995, ApJ, 455, L109

Weisskopf, M. C., O’Dell, S. L., \& Van Speybroeck, L. P. 1996, Proc. SPIE, 2805, 2

Yang, Y., Mushotzky, R. F., Barger, A. J., et al. 2003, ApJ, 585, L85

Zappacosta, L., Mannucci, F., Maiolino, R., et al. 2002, A\&A, 394, 7 